\title{
Philosophiques
}

\section{L'institutionnalisation de la raison publique : le moral et le politique}

\section{Jocelyne Couture}

Volume 24, numéro 1, printemps 1997

Avez-vous lu Rawls?

URI : https://id.erudit.org/iderudit/027423ar

DOI : https://doi.org/10.7202/027423ar

Aller au sommaire du numéro

\section{Éditeur(s)}

Société de philosophie du Québec

ISSN

0316-2923 (imprimé)

1492-1391 (numérique)

Découvrir la revue

Citer cet article

Couture, J. (1997). L'institutionnalisation de la raison publique : le moral et le politique. Philosophiques, 24(1), 43-57. https://doi.org/10.7202/027423ar
Résumé de l'article

La question de l'allégeance morale au libéralisme politique occupe une place centrale dans la théorie de John Rawls. J'arguerai d'abord que, dans la conception de Rawls, le moral et le politique entretiennent un rapport paradoxal qui risque de compromettre l'institutionnalisation de la raison publique telle que Rawls la conçoit. Je proposerai ensuite une nouvelle définition des conditions de l'allégeance morale au libéralisme politique. J'arguerai que ma définition, tout en évitant la tension relevée chez Rawls entre le politique et le moral, est aussi mieux adaptée aux représentations contemporaines du citoyen en tant qu'agent moral et plus conforme aux intuitions courantes concernant le rôle de la moralité dans une démocratie constitutionnelle. 


\title{
L'INSTIIUUIIONNALISATION DE LA RAISON PUBLIQUE : IE MORAL ET LE POLITIQUE
}

\author{
PIR \\ JOCELYNE COUIURE
}

\begin{abstract}
RÉSUMÉ: La question de l'allégeance morale au libéralisme politique occupe une place centrale dans la théorie de John Rawls. J'arguerai d'abord que, dans la conception de Rawls, le moral et le politique entretiennent un rapport paradoxal qui risque de compromettre l'institutionnalisation de la raison publique telle que Rawls la conçoit. Je proposerai ensuite une nouvelle définition des conditions de l'allégeance morale au libéralisme politique. J'arguerai que ma définition, tout en évitant la tension relevée chez Rawls entre le politique et le moral, est aussi miewx adaptée aux représentations contemporaines du citoyen en tant qu'agent moral et plus conforme aux intuitions courantes concernant le rôle de la moralité dans une démocratie constitutionnelle.
\end{abstract}

ABSTRACT: The problem of the moral allegiance to political liberalism is central in the theory of John Rawls. It will be argued first, that, as Rawls conceive them, the moral and the political practically stand in a paradoxical relationship which undermines the possibilities for the implementation of " public reason". A new characterization of the conditions of moral allegiance will be articulated. I will then argue that my characterization removes the tensions between the moral and the political I noticed in Rawls. It also yields a better understanding of the actual representation of the citizen as a moral agent and finally, gives a more accurate account of the role of morality in contemporary constitutional democracies.

Etre raisonnable, c'est agir pour des raisons. Et ètre publiquement raisonnable, c'est agir publiquement, pour des raisons publiques, c'est-à-dire des raisons connues de chacun et acceptées par chacun de ceux à qui il importe d'agir pour des raisons. Dans une société pluraliste, les raisons, publiques dans ce sens, ne peuvent pas dépendre de doctrines philosophiques, métaphysiques, morales ou religieuses qui, par définition, ne sont pas acceptées par chacun de ceux à qui il importe d'agir pour des raisons. Par conséquent, les actions, les décisions et les arrangements raisonnables dans le domaine public ne dépendront pas non plus de telles doctrines. 
Plusieurs des auteurs qui ont fait usage de la notion de raison publique en philosophie politique ${ }^{1}$ l'ont fait dans un contexte de justification, c'est-à-dire dans un contexte où il importait de montrer que leur conception de l'organisation politique pouvait en principe satisfaire aux exigences de la raison publique, mais où il importait peu, finalement, de dresser la liste des raisons capables de témoigner du caractère raisonnable de leur conception.

Dans un contexte de justification, la notion de raison publique soumet une conception de l'organisation politique à deux exigences. La première est qu'une telle conception soit démocratique au sens où elle ne tient pour légitimes que des arrangements publics conclus sur la base des raisons comprises et acceptées des citoyens. La deuxième est qu'une conception politique soit libérale au sens où, recommandant l'autonomie du politique vis-à-vis des doctrines - religieuses, morales ou autres - entretenues par les citoyens, elle reconnait le pluralisme et préconise la tolérance. En posant ces deux exigences, la notion de raison publique désarticule le politique et le moral. Les conceptions communes - et les doctrines morales ne peuvent pas l'être dans une société pluraliste - constituent le seul fondement admissible des institutions et des arrangements politiques. Une constitution conçue, dans un pur contexte de justification, comme un produit de la raison publique n'a pas d'assise morale.

Contrairement aux autres penseurs de la raison publique, John Rawls spécifie le contenu de celle-ci ; il dresse une liste des raisons qui, dans une société pluraliste, sont de nature à asseoir des arrangements politiques raisonnables, c'est-à-dire légitimes. Ce faisant, Rawls quitte le terrain de la justification d'une conception politique normative pour s'avancer sur celui de l'explicitation de la norme politique valide en vertu de cette conception. Il assigne à la raison publique une fonction additionnelle : celle de définir ce que j'appellerai "la pratique citoyenne", c'est-à-dire la pratique politique du citoyen d'une démocratie constitutionnelle.

Dans cet article, je me propose d'abord de montrer qu'en caractérisant ainsi la notion de raison publique, Rawls en fait l'instrument principal d'une réarticulation, au plan conceptuel, du rapport entre le politique et le moral; de ce rapport, en particulier, auquel on fait allusion lorsque l'on évoque, plus ou moins obscurément, l'allégeance morale que devrait susciter une constitution de type libéral. J'arguerai ensuite qu'au plan pratique - là où devrait s'instaurer une pratique citoyenne - , le moral et le politique entretiennent un rapport paradoxal qui risque de compromettre l'institutionnalisation de la raison publique telle que Rawls la conçoit. Dans la dernière partie de cet article, je proposerai une nouvelle définition des conditions de l'allégeance morale au libéralisme politique. J'arguerai que ma définition, tout en évitant la tension relevée chez Rawls entre le politique et le moral, est aussi mieux adaptée aux représentations contemporaines du citoyen en tant quagent moral et plus conforme aux intuitions courantes concernant le rôle de la moralité dans une démocratie constitutionnelle.

1. Pour un exposé détaillé, voir Onora O'Neill, Constructions of Reason, Cambridge, Cambridge University Press, 1989. 


\section{Le concept rawlsien de raison publique \\ Le contenu de la raison publique}

Les arrangements politiques raisonnables sont ceux qu'appuient des raisons publiques, c'est-à-dire des raisons connues de chacun et acceptées par chacun de ceux à qui il importe d'agir pour des raisons. Quelles sont, dans une démocratie constitutionnelle contemporaine, les raisons capables de rendre raisonnables les arrangements politiques? La liste que donne Rawls n'est pas très longue et elle est comprise dans ce qu'il appelle "le contenu de la raison publique $^{2}{ }^{*}$.

Il s'agit, premièrement, de deux principes de justice politique applicables à la structure de base d'une société ${ }^{3}$ et, deuxièmement, des valeurs politiques qui sous-tendent ces principes, à savoir l'égalité des libertés civiles et politiques, la réciprocité économique, l'égalité des chances et l'égalité sociale. Ces principes de justice et ces valeurs peuvent ètre vus comme des raisons au sens où ils forment la base légitimante des dispositions constitutionnelles qui spécifient les libertés et les droits fondamentaux des citoyens, de mème que la forme des principales institutions de base d'une société démocratique. Ils constituent aussi la base légitimante des décisions des pouvoirs législatif et exécutif qui interprètent la constitution ainsi que des arrangements requis par elle et liés aux inégalités sociales et économiques.

Font aussi partie de la raison publique ce que Rawls nomme les lignes directrices de l'enquête. Il s'agit d'abord des principes de raisonnement et ensuite des règles d'évidence qui spécifient les conditions et les circonstances de l'application des principes de justice et qui permettent de déterminer les politiques qui les satisfont le mieux. Ces lignes directrices de l'enquête s'accompagnent aussi de valeurs politiques telles que la liberté et la publicité de l'enquête, la volonté d'honorer ses devoirs de citoyens et de pratiquer les vertus qui rendent possible la discussion publique. Les lignes directrices de l'enquēte et les valeurs politiques qui les sous-tendent sont des raisons, tout comme l'existence d'une démonstration, conforme à des techniques et à des standards généralement acceptés, est une raison d'affirmer une proposition qui en serait la conclusion. Avec les principes de la justice et les valeurs politiques qui les accompagnent, elles constituent l'argumentaire public d'où les arrangements politiques doivent tirer leur légitimité.

2. John Rawls, Political Liberalism New York, Columbia University Press, 1993, p. 224.

3. Par structure de base, Rawls entend " [...] a society's main political sociol, and economic institutions, and how they fit together into one unified system of social cooperation from one generation to the next. "Ibid., p. 11. Les deux principes sont, à peu de choses près, ceux que l'on retrouvait déjá dans $\mathrm{J}$. Rawls. A Theory of Justice, Harvard University Press, 1971 : «[...] a) Each person has an equal right to the most extensive scheme of equal basic liberties compatible with a similar scheme of liberties for all. b) Social and economic inequalities are permissible provided that they are i) to the greatest expected benefit of the least advantaged : and ii) attached to positions and offices open to all under conditions of fair equality of opportunity. " J. Rawls, Political Liberalism, p. 271. 


\section{L. pratique citoyenne}

En arrêtant la liste des raisons publiques, Rawls énonce les normes appelées à régir le forum politique. Il fait de la raison publique la raison du public; celle des citoyens d'une démocratie constitutionnelle, non seulement autorisés à prendre part aux décisions, mais aussi juges et gardiens des processus par lesquels on $y$ arrive. Les normes de la raison publique circonscrivent une pratique citoyenne ${ }^{4}$. En tant qu'exécuteur de la raison publique, le citoyen n'accepte aucun arrangement politique qui ne soit clairement établi à partir des raisons qu'énonce la raison publique. Et il se conforme aux exigences de la raison publique; lorsqu'il intervient dans la sphère publique, que ce soit pour contester ces arrangements, pour en proposer ou pour voter ${ }^{5}$, les raisons auxquelles il fait appel sont celles qui sont admissibles dans le domaine public; ce sont les raisons publiques. Nulle autre raison relative à ses propres valeurs, à ses intérêts ou objectifs personnels ne ferait de ses interventions dans le domaine politique des interventions raisonnables.

Ainsi définie par Rawls, la raison publique remplit trois fonctions. Premièrement, elle est constitutive de la démocratie puisqu'elle garantit aux citoyens une libre et égale participation aux décisions politiques. Deuxièmement, elle consacre l'autonomie du politique en excluant de ce domaine toute considération qui dépendrait de doctrines particulières, qu'elles soient morales, métaphysiques, religieuses ou philosophiques. Les citoyens entretiennent de telles doctrines, que Rawls appelle des conceptions compréhensives du bien ${ }^{6}$, mais dans une société pluraliste ces conceptions sont sujettes à controverses et ne fournissent, par conséquent, aucune raison admissible dans la sphère politique ${ }^{7}$. Et troisièmement, la raison publique énonce les normes qui instaurent et définissent la pratique citoyenne. En stipulant les règles selon lesquelles le citoyen doit s'acquitter de ses devoirs et de ses attributions au sein du forum démocratique. elle fait de lui l'exécuteur même de la raison publique.

4. Public reason is characteristic of a democratic people; it is the reason of its citizens, of those sharing the status of equal citizenship. [...] That public reason should be so understood and honored by citizens is not, of course, a matter of law. As an ideal conception of citizenship for a constitutional democratic regime, it presents how things might be, taking people as a just and well-ordered society would encourage them to be. "Ibid., P. 213.

5. "On fundamental political questions the idea of public reason rejects common views of voting as a private and even personal matter. " Ibid., p. 219.

6. "A moral conception [...] is comprehensive when it includes conceptions of what is of value in human life, and ideals of personal character, as well as ideals of friendship and of familial and associational relationships, and much else that is to inform our conduct, and in the limit to our life as a whole. (...) Utilitarianism is a familiar example (of comprehensive views): the principle of utility, however understood, is usually said to hold for all kinds of subjects ranging from the conduct of individual and personal relations to the organization of society as whole as well as to the law of peoples. "Ibid. p. 13.

7. " $[. .$.$] the question the dominant tradition has tried to answer has no answer : no$ comprehensive doctrine is appropriate as a political conception for a constitutional regime. "Ibid, p. 135. 


\section{Le citoyen et sa raison}

Comme la plupart des penseurs de la raison publique, Rawls croit qu'une constitution qui satisfait aux exigences de la raison publique, dans ses deux premières fonctions, est une constitution justifièe d'un point de vue libéral. Et il pense sans nul doute qu'une constitution ainsi justifiée sera justifiée aux yeux des citoyens habitués à vivre dans le cadre des institutions politiques d'une démocratie libérale. Mais Rawls pense aussi que la stabilité des institutions démocratiques dépend de l'application soutenue, et constamment réitérée, des normes de la raison publique qui définissent la pratique citoyenne ${ }^{8}$. Pourquoi Rawls croit-il que les citoyens d'une démocratie constitutionnelle accepteront de se conformer à cette pratique citoyenne, pourquoi croit-il que les citoyens s'acquitteront des devoirs et des obligations qu'elle leur impose?

La réponse de Rawls s'articule à plusieurs niveaux, mais ce qui est au cour de sa réponse est l'idée que, du point de vue de leur conception compréhensive du bien, les citoyens considèrent qu'une société régie par la raison publique est un bien. Dans ses deux premières fonctions, la raison publique garantit cette sorte de société à laquelle chacun aspire, c'est-à-dire, selon Rawls, une société qui offre à chacun une opportunité égale de poursuive et de réaliser sa propre conception compréhensive du bien. Mais en rendant explicites les normes de la vie en société, la raison publique réussit de plus à éliminer les principales sources des incompréhensions mutuelles et des dissensions qui mettent en péril les interactions sociales et, par conséquent, les avantages que chacun attend de la vie en société. Une société régie par la raison publique telle que Rawls la définit est une société où l'on a, selon lui, réglé une fois pour toutes, au vu et au su de chacun, la question litigieuse par excellence du sens et de l'extension des concepts d'égalité et de liberté $\dot{e}^{9}$. C'est une société où règne donc un minimum d'entente sur des questions fondamentales, mais aussi sur la manière de régler les problèmes qui découlent de ces questions. C'est aussi une société où chacun connait, et sait que tous connaissent, les exigences ainsi que les limites de la tolérance. Bref, la raison publique, parce qu'elle énonce clairement les règles, et non seulement les principes, de la participation démocratique, rend possible la coordination des comportements ; elle encourage la pratique de l'amitié civique et conduit ainsi à la réalisation du type de société qui permet à chacun de poursuivre ses propres fins conformément à ses valeurs et à ses intérèts. Dans cette optique, respecter et faire respecter les normes de la raison publique constitue moins une contrainte pour le citoyen qu'un moyen de maximiser ce qui, selon Rawls, semble ètre l'avantage majeur que présente pour le

8. The safety of democratic liberties requires the active participation of citizens who possess the political virtues needed to maintain a constitutional regime. "Ibid, p. 205.

9. "The first principles (of justice) are used to settle the appropriate understanding of freedom and equality for a modern democratic society ". J. Rawls, "Kantian Constructivism in Moral Theory ". The Joumal of Philosophy, $\mathrm{n}^{\circ} 7, \mathrm{p}$. 554. *...this conflict of understandings sets the present practical task of political philosophy *, ibid., p. 538. 
citoyen la vie en société, à savoir la réalisation de sa conception compréhensive du bien.

La réponse de Rawls à la question de l'adhésion à la pratique citoyenne est ainsi une réponse qui présuppose que chacun entretienne une conception compréhensive du bien ${ }^{10}$. Et en effet, Rawls soutient que la personne idéale - celle qui désire ce type de société et celle que produit ce type de société - est une personne morale, caractérisée par le fait de posséder un sens de la justice et une conception du bien et guidée, dans tout le cours de sa vie, par la volonté de réaliser l'un et l'autre ${ }^{11}$. Voici donc un premier argument qui rend l'adhésion aux normes de la raison publique et, par conséquent, la stabilité des institutions démocratiques, conditionnelles au fait que les personnes possèdent une conception compréhensive du bien.

Un deuxième argument qui avalise et renforce en fait cette condition est celui dans lequel Rawls soutient que chacun trouve, dans sa conception compréhensive particulière du bien, les motifs de souscrire à la raison publique, aux valeurs politiques qu'elle met de l'avant et aux règles de la participation démocratique qu'elle impose aux citoyens. Il ne s'agirait plus simplement ici de voir, dans la société régie par la raison publique, un bien instrumental, mais de reconnaître dans la société politique une partie constituante de son propre bien ${ }^{12}$. L'adhésion à la raison publique serait conditionnelle à la valeur que chacun peut assigner à ses contenus du point de vue de sa conception compréhensive du bien ${ }^{13}$.

Ainsi, le citoyen accepterait de se faire l'exécuteur de la raison publique parce que celle-ci, premièrement, produit ce type de société qui lui permet de réaliser sa propre conception compréhensive du bien et, deuxièmement, fait appel à des valeurs qui font partie de sa propre conception compréhensive du bien. Une constitution libérale, conçue comme le produit de la raison publique, pourrait, après tout, susciter l'allégeance morale des citoyens. Mais ce que requiert d'eux la pratique citoyenne est justement de ne pas chercher à faire valoir et, a fortiori, de ne pas chercher à réaliser leurs conceptions morales au sein de l'espace politique.

\section{L'institutionnalisation de la rais on publique}

Un dil em me

On peut supposer, tout comme le fait Rawls, qu'il est possible pour des individus, en leur qualité d'agents moraux, d'accorder à la société politique une valeur instrumentale. On peut a fortiori supposer qu'il est possible, pour de tels individus, de travailler activement,

10. Il s'agit par ailleurs d'une position que Rawls affirme généralement et pas seulement dans le contexte de cet argument. Voir, par exemple, J. Rawls, Political Liberalism p. XIX, 12, 38, 135, 140, 150, 175.

11. Voir à ce sujet ibid., p. 201-204. Rawls insiste sur le fait que la conception de la personne qu'il suppose ici doit ètre comprise " as a political conception and not as one belonging to a comprehensive doctrine *, p. 178.

12. Ibid., p. $201-204$.

13. "Citizens affirm the ideal of public reason, not as a result of political compromises, as in a modus vivendi but from within their own reasonable doctrine. "Ibid., p. 218. 
en leur qualité d'agents rationnels, à l'édification et au maintien de ce type d'organisation politique ; en souscrivant aux normes de la pratique citoyenne, ils ne font qu'agir selon la conception morale qui fonde en premier lieu la valeur instrumentale que revèt pour eux une société de ce type. On peut de plus supposer, tout comme le fait encore Rawls, que des agents moraux trouvent, dans leur conception compréhensive du bien, des motifs de souscrire aux normes de la raison publique. Mais il n'y a aucune raison de supposer que les agents moraux ne trouveront pas, dans leur conception compréhensive du bien, des motifs peut-être plus importants pour eux de souscrire à une pratique citoyenne bien différente de celle que propose Rawls. Si leur conception compréhensive du bien est pertinente lorsqu'il s'agit de reconnaître le bien-fondé de la pratique citoyenne rawlsienne, elle devrait aussi l'ètre lorsqu'il s'agit de définir autrement les devoirs du citoyen. Pourquoi, dans de tels cas, des individus rationnels se conformeraient-ils à la pratique citoyenne rawlsienne qui, tout en reconnaissant la pertinence de leurs conceptions morales, leur demande de ne pas faire intervenir les raisons morales qui, à leurs yeux, motivent le mieux une pratique citoyenne? Pourquoi accepteraient-ils de se conformer à une pratique citoyenne qui, selon eux, est ainsi entachée d'arbitraire moral; et pourquoi devraient-ils accepter que leurs conceptions compréhensives n'aient qu'une valeur instrumentale dans le maintien d'une société régie par la raison publique. Pourquoi, bref, des individus moraux accepteraient-ils de faire abstraction de leur conception compréhensive lorsqu'ils travaillent à l'édification d'une société qu'ils évaluent moralement ? C'est pourtant ce qu'exige la raison publique dans tous les cas où les conceptions morales des citoyens incluent davantage que les valeurs impliquées par les normes de la raison publique. Et telle est, sous diverses formulations, la prémisse du raisonnement de Rawls : les conceptions morales qui fondent l'allégeance des citoyens au libéralisme politique sont des conceptions compréhensives qui incluent d'une façon ou d'une autre, mais ne s'y limitent pas, les valeurs impliquées par les normes de la raison publique ${ }^{14}$.

Plusieurs critiques ${ }^{15}$ de Rawls ont souligné que les conceptions compréhensives que Rawls place au fondement de l'adhésion aux normes de la raison publique risquent de faire obstacle à la pratique de la raison publique : il s'agit d'une objection sérieuse puisque Rawls soutient, comme nous l'avons vu, que la pratique citoyenne est elle-même une condition nécessaire à la survie des libertés démocratiques. Du point de vue qui nous intéresse ici, ce problème reçoit une autre formulation: l'allégeance morale à la société politique serait

14. "I assume all citizens to affirm a comprehenswe doctrine to which the political conception they accept is in some way related (...) The political conception is a module, an essential constituent part, that fits into and can be supported by various reasonable comprehensive doctrines that endure in the society regulated by it. " Ibid., p. 12.

15. Voir, par exemple, Joshua Cohen, "Democratic Equality ", Ethics 99, 1989, p. 727-751 et "A more Democratic Liberalism ", Michigan Law Review 92, $\mathrm{n}^{\circ}$ 6, 1994, p. 1503-1546 ; Jean Hampton, "Should Political Philosophy be Done without Metaphysics *, Ethics 99, 1989, p. 791-814 et Richard Rorty, Objectivity, Relativism and Thruth, Cambridge University Press, 1991, p. 175-197. 
cela même qui s'oppose à la réalisation et au maintien de ce type de sociétè.

La tension entre ce que doit croire le citoyen pour adhèrer au libéralisme politique et ce que requiert de lui la pratique citoyenne pourrait aussi se manifester dans le sens inverse, avec un résultat similaire et tout aussi dommageable pour ce qui est du rapport entre le moral et le politique. Supposons un instant que, en dépit des obstacles auxquels nous venons de faire allusion, la raison publique rawlsienne parvienne à s'imposer auprès des citoyens. Que la raison publique parvienne à s'imposer implique que le citoyen admette que la conception morale qui, dans sa vie privée, est la source de ses raisons d'agir, devient la source de mauvaises raisons dans le domaine politique. S'il croit cela, comment peut-il continuer d'entretenir la conception compréhensive qui fonde ses "mauvaises " raisons politiques? Une conception compréhensive, selon la définition de Rawls, consiste en une doctrine visant à gouverner l'ensemble de la vie et ceux qui y adhèrent la croient vraie. L'individu qui se conforme à la pratique citoyenne devrait croire que sa conception compréhensive est vraie, bien qu'elle soit la source de mauvaises raisons politiques. Il parait plus plausible de supposer que puisqu'il la juge non applicable dans le domaine politique, le citoyen la tiendra pour partiellement vraie et en viendra à l'abandonner en tant que conception compréhensive. La pratique citoyenne conduirait ainsi à l'érosion des conceptions compréhensives ${ }^{16}$. L'usage de la raison publique saperait ainsi sa propre base, c'est-à-dire la base morale à partir de laquelle le citoyen est censé accepter de s'y conformer. Et la raison publique ruinerait du même coup les conditions de stabilité, telles qu'elles sont conçues par Rawls, des institutions de type libèral.

Ou bien les conceptions compréhensives prennent le pas sur la raison publique ou bien c'est l'inverse : la raison publique prend le pas sur les conceptions compréhensives. Les conséquences sur les conditions de stabilité des institutions démocratiques, telles qu'elles sont conçues par Rawls, sont les mèmes dans l'un et l'autre cas. Et les conséquences sur la réconciliation du moral et du politique sont également les mêmes dans l'un et l'autre cas. Le politique, conçu comme un achèvement de la raison publique, entretient des rapports

16. Un passage de Political Liberalism illustre cette difficulté. Dans ce passage. Rawls explique que l'éducation religieuse est tout à fait admissible dans une société liberale à la condition que * children's education include such things as knowledge of their constitutional and civic rights so that for example, they know that liberty of conscience exists in their society and that apostasy is not a legal crime [... " " Political Liberalism p. 199. Rawls nie qu'une telle éducation civique ait comme résultat l'* inculcation * d'une conception libérale comprêhensive. Mais il semble admettre qu'elle puisse avoir un effet corrosif sur la foi religieuse. "The unavoidable consequences of reasonable requirements for children's education may have to be accepted, often with regret *. p. 200. Mon argument ici est que les normes de la raison publique qui exigent des individus que, dans la pratique, ils suspendent leurs conceptions morales ont, sur celles-ci, les mêmes effets corrosifs. Si tel est le cas, Rawls a tort de penser que le pluralisme est là pour durer, mais il a aussi tort de penser que les conditions de stabilité des institutions politiques peuvent être liées à des conceptions morales compréhensives. 
paradoxaux avec cela mème qui devrait confirmer l'allégeance morale des citoyens à leurs institutions politiques.

\section{Le moral et le politique : doit-on choisir?}

L'adhésion à la raison publique c'est, entre autres choses, l'adhésion à un principe de séparation du politique et du moral et ce principe, comme on le sait, est un pilier du libéralisme. L'autre pilier du libéralisme consiste en un principe de participation démocratique à la vie politique. L'adhésion à ce principe est aussi impliquée par l'adhésion à la raison publique. En faisant de la raison publique la norme d'une pratique citoyenne, Rawls fait du citoyen l'exécuteur de ces deux principes. La participation à la vie démocratique et la pratique de la tolérance deviennent les devoirs que le citoyen doit respecter et faire respecter. Rawls soutient de plus que c'est en vertu de sa conception compréhensive du bien que le citoyen voit qu'il y va de ses devoirs de respecter et de faire respecter les normes de la raison publique. Comme nous venons de le voir, la base morale requise par Rawls entre en conflit avec les exigences pratiques de la raison publique telles que Rawls les définit. Pour le dire autrement, si la participation démocratique requiert du citoyen ce qu'elle requiert en vertu de la pratique citoyenne et si sa base motivationnelle doit de plus résider dans des conceptions compréhensives du bien, alors les deux piliers du libéralisme mettent en place, pour le citoyen, des exigences contradictoires.

Parvenu à ce point, on pourrait se demander si l'idée de l'allégeance morale à une constitution de type libéral et fruit de la raison publique n'est pas elle-mème porteuse de ses propres contradictions. Rawls semble plutōt croire le contraire, à savoir que le projet du libéralisme politique sans allégeance morale est un projet inconsistant.

J'ai fait allusion plus tôt à l'argument par lequel Rawls établit que la stabilité des institutions démocratiques libérales dépend d'une pratique citoyenne ancrée dans les convictions morales des individus. Le corollaire de cet argument amène Rawls à conclure que la stabilité des institutions démocratiques libérales dépend de l'existence d'un consensus sincère au sein de la société, c'est-à-dire d'un consensus qui trouve son fondement dans les conceptions compréhensives du bien entretenues par les citoyens ${ }^{17}$. Sans allégeance morale, le libéralisme politique ne possède, selon Rawls, aucune chance de survie. Il risquerait même de se transformer en son contraire, si l'on se fie à une objection récemment adressée par Rawls à Habermas ${ }^{18}$. En vertu de cette objection, en effet, une conception politique destinée, comme celle de Habermas, à supplanter les conceptions morales au sein d'une société devient par le fait mème une

17. Voir à ce sujet Jocelyne Couture, "Pourquoi devrait-il y avoir un conflit entre le nationalisme et le libéralisme politique? n, dans $F$. Blais, D. Lamoureux et G. Laforest (éd.), Libéralismes et nationalismes, P.U.L.. 1995, p. $51-75$.

18. J. Rawls, "Reply to Habermas ". The Journal of Philosophy, $n^{\circ}$ 3, 1995, p. 132-180. 
conception compréhensive ${ }^{19}$. $\mathrm{Si}$, comme nous en avons fait l'hypothèse, le libéralisme conduit à l'érosion des conceptions compréhensives entretenues par les citoyens, ce serait pour s'imposer lui-mème en tant que conception compréhensive. Mais en tant que tel, ce "libéralisme" ne souscrirait ni à un principe de tolérance ni au pluralisme.

Sur la voie tracée par Rawls, il n'y a, semble-t-il, aucune issue au dilemme de l'institutionnalisation de la raison publique.

\section{La raison publique et la moralité de l'individu libéral}

\section{Le citoye $n$ et sa conception du bien}

L'idèe qu'une constitution libèrale, conçue sur le modèle de la raison publique, puisse susciter l'allégeance morale des citoyens semble donc conduire à une impasse. Si l'allégeance morale doit faire appel, comme chez Rawls, aux conceptions compréhensives du bien qu'entretiennent les citoyens, alors celles-ci ou bien font obstacle à la pratique de la raison publique, ou bien disparaissent sous son effet. Dans le premier cas, les motifs d'une allégeance morale ne sont pas réunis et, dans le second cas, ce sont les sources morales de l'allégeance qui font défaut. Par ailleurs si, comme le suggère la réponse de Rawls à Habermas, l'allégeance morale trouve sa source exclusive dans la raison publique elle-même, c'est que celle-ci représente une conception compréhensive. Mais puisque par définition, aucune conception compréhensive ne peut ètre compatible avec toutes celles que peuvent entretenir les citoyens d'une société pluraliste, il s'ensuit que la raison publique, dans ces conditions, n'est la raison ni d'une société tolérante ni d'une sociēté pluraliste ; elle est, comme nous venons de le voir, l'équivalent fonctionnel d'une unique conception compréhensive du bien. Elle cesse dès lors de satisfaire aux exigences du libéralisme politique qu'elle devrait pourtant représenter.

Ou bien la raison publique est libérale et alors, comme nous l'avons vu, elle risque fort de ne pas susciter une allégeance morale, ou bien elle suscite l'allégeance morale, mais cesse d'être libérale puisqu'elle représente alors une conception compréhensive du bien. Mais une allégeance de nature morale, susceptible de garantir la stabilité des institutions démocratiques, doit-elle absolument, comme le croit Rawls, ètre à ce point liée à une conception compréhensive du bien?

L'idée que les citoyens d'une démocratie constitutionnelle entretiennent des conceptions comprẻhensives du bien est indissociable de la conception que se fait Rawls du pluralisme dans les démocra-

19. Une objection similaire est formulêe par Rawls à l'endroit des conceptions ou் " participation (in political life) is not encouraged as necessary for the protection of the basic liberties of democratic citizenship [...] Rather, taking part in democratic politics is seen as the privileged locus of the good life. "J. Rawls, Political Liberalism p. 206. Rawls attribue une telle doctrine à Aristote et, selon lui, il s'agit d'une conception comprẻhensive du politique. Comme me le rappelle fort justement un lecteur anonyme d'une première version de cet article, Rawls, dans Political Liberalism, considère aussi que le libéralisme de John Stuart Mill, tout comme celui de Will Kymlicka, constituent des conceptions compréhensives du bien. 
ties contemporaines. Selon Rawls, une multitude de valeurs mutuellement exclusives cohabitent dans nos sociétés contemporaines et ces valeurs sont de plus organisées dans des doctrines compréhensives, ce qui contribue encore, si cela est possible, à l'incommensurabilité des conceptions morales ${ }^{20}$.

Les idées communes, appelées à faire partie d'une raison publique authentiquement libérale, en sont d'autant plus rares. Non seulement ces idèes doivent ètre communes, c'est-à-dire, partagées par tous les citoyens "raisonnables ", mais elles doivent de surcroit être compatibles avec des ensembles consistants de valeurs qui constituent les conceptions compréhensives du bien entretenues par les citoyens. Il en résulte que pour se conformer à la pratique citoyenne, chacun est requis de tenir en suspens, dans le domaine politique, des fragments considérables de sa conception compréhensive du bien qu'il juge par ailleurs essentiels à ses conceptions morales. Tel est le dilemme dont nous avons parlé dans la section précédente: ou bien les conceptions font obstacle à la pratique citoyenne ou bien cette dernière conduit à l'érosion des valeurs morales.

Est-ce bien le cas que le pluralisme, dans les sociétés contemporaines, prenne la forme de cette opposition profonde et quasi irréductible des conceptions de la vie bonne? Est-ce bien le cas, premièrement, qu'une caractéristique des citoyens des démocraties constitutionnelles est d'entretenir une conception compréhensive du bien ? Je voudrais suggérer ici que la tension qui existe chez Rawls entre les conditions de l'allégeance morale et la pratique citoyenne telle qu'il la définit est en fait une tension entre cette dernière et une conception insuffisamment libérale de la personne.

\section{L'individu libéral et ses conceptions du bien}

Le citoyen d'une démocratie constitutionnelle est un individu qui a développé une familiarité avec un certain type d'organisation et de fonctionnement politique. Sans prétendre qu'il en a intériorisé les principes, on peut néanmoins supposer qu'il a pris l'habitude de s'y conformer. C'est aussi un individu qui a développé une familiarité avec les principales institutions politiques et économiques et avec les principaux types d'associations caractéristiques des sociétés démocratiques contemporaines. Sans devoir prétendre qu'il en approuve la forme ou le fonctionnement actuels, on peut néanmoins supposer qu'il reconnait la fonction de coordination sociale dévolue à ces institutions.

Le citoyen d'une démocratie constitutionnelle reconnait que des normes diverses régissent différents aspects de sa vie en société et il reconnait que chacun de ces aspects doit, pour le bon ordre de la société, ètre régi par des normes spécifiques et appropriées. En ce qui a trait à la nature exacte de ces normes, il entretient probablement ses propres vues. Il entretient, en tous cas, des conceptions

20. "A modern democratic society is characterized not simply by a pluralism of comprehensive religious, philosophical and moral doctrines but by a pluralism of incompatible yet reasonable (je souligne) comprehensive doctrines ", ibid. p. XVI. 
de ce que doivent être sa vie professionnelle, sa vie familiale, sa vie politique ; il a identifié les fins spécifiques qu'il importe de poursuivre, selon lui, dans chacun de ces domaines et les ressources particulières sur lesquelles il doit pouvoir compter dans chaque cas pour réaliser ces objectifs.

Chacune de ces conceptions est une conception du bien au sein de laquelle les valeurs morales jouent, sans nul doute, un rôle décisif. Mais d'une conception à l'autre, ces valeurs morales n'ont besoin, ni d'être identiques, ni d'être complémentaires, ni même de former un système consistant. Les diverses conceptions du bien qu'entretient simultanèment le citoyen d'une démocratie constitutionnelle contemporaine n'ont pas besoin de s'organiser - et ne s'organisent généralement pas - en une conception compréhensive du bien. Elles ne sont pas des fragments d'une doctrine générale et unitaire dont il croirait qu'elle peut gouverner tous les aspects de la vie.

À l'échelle des sociétés contemporaines, plusieurs conceptions du bien cohabitent et certaines, bien sür, sont des conceptions compréhensives. Mais la plupart des individus habitués aux modes de vie contemporains entretiennent simultanément plusieurs conceptions du bien s'appliquant respectivement à des domaines différents de leur vie. Le pluralisme est au sein de chaque personne et chacune pratique la tolérance à l'endroit de ses propres conceptions lorsque, dans un domaine donné, elles s'avèrent non pertinentes.

\section{Les nouvelles exigences de la raison publique}

Si le pluralisme est au sein de chaque personne au sens où je viens de le dire, il y a bien un autre moyen que celui qu'envisagent Rawls et les penseurs de la raison publique de s'assurer de l'autonomie du politique dans une société libérale. C'est de concevoir les raisons communes, connues et acceptées de tous à partir des conceptions $d u$ politique qu'entretiennent les individus, sans faire appel aux conceptions qu'ils entretiennent aussi du familial, du professionnel ou du religieux.

Et si le pluralisme est en chacun de nous au sens où je viens de le dire, il y a bien une façon de réunir le moral et le politique. C'est, encore une fois, de prendre en considération les conceptions du politique qu'entretiennent les individus d'une société libérale; ces conceptions sont hétérogènes, comme je viens de le dire, et elles incluent des valeurs morales, pertinentes dans le domaine politique, mais qui n'ont pas nécessairement à l'être dans d'autres domaines.

$\mathrm{Si}$ les raisons communes sont définies à partir des conceptions du politique qu'entretiennent les citoyens, et que ces conceptions, comme je viens de le suggérer, incluent des valeurs morales pertinentes pour le domaine de lorganisation politique, alors la raison publique fait appel à des valeurs morales. Il n'en faut pas plus, je pense, mais pas moins non plus, pour que l'on puisse parler de l'allégeance morale au libéralisme politique et à ses institutions.

Les normes de la raison publique. lorsqu'elles sont ainsi conçues, risquent-elles encore d'imposer aux citoyens des exigences contradictoires? 
Elles requièrent des citoyens qu'ils ne fassent pas intervenir, dans le domaine politique, leurs conceptions non politiques. Elles requièrent aussi des citoyens qu'ils tiennent en suspens, dans le forum public, certains fragments de leurs conceptions politiques. Dans une société pluraliste, les valeurs et les idées constitutives de ces conceptions ne sont pas toutes communes; il n'y a aucune raison de croire, cependant, que les valeurs morales ont moins de chance que les autres valeurs d'être communément partagées. Par ailleurs, une idée qui est commune, dans une société formée d'individus pluralistes dans leur conception du bien, est l'idée que les conceptions du bien politique, tout comme les autres conceptions du bien qu'ils entretiennent, font intervenir des valeurs morales. La raison publique n'a plus à exiger des citoyens que dans le domaine politique ils mettent en veilleuse, au nom de la tolérance, un type de considérations qui, dans quelque domaine que ce soit, revêt une importance cruciale pour eux, à savoir les considérations de nature morale. La raison publique n'a plus à requérir du citoyen que, dans le forum politique, il cesse d'être moral. Au contraire, la participation démocratique, définie par les normes de la raison publique, inclura la pratique de certaines vertus et la promotion de valeurs perçues par les citoyens comme étant des vertus et des valeurs proprement morales et perçues par chacun comme étant des valeurs communes.

Ces vertus et ces valeurs perçues comme morales n'ont pas à ètre extensionnellement différentes, et ne le sont peut-être pas dans une démocratie constitutionnelle contemporaine, de ce que Rawls appelle les valeurs de la justice politique. Mais elles pourraient aussi être plus nombreuses, puisqu'elles n'ont plus à s'ancrer dans des systèmes de valeurs dont l'étendue fait croitre les risques d'incommensurabilitée ${ }^{21}$. Ainsi, même si le contenu de la raison publique rawlsienne devait s'enrichir de quelques valeurs morales, sa pratique n'en demeurerait pas moins conforme aux exigences de la tolérance dans une société pluraliste.

L'hypothèse que le pluralisme est aussi au sein des personnes respecte donc l'intégrité de la conception rawlsienne de la société politique libérale et du rōle que peut y jouer la raison publique. Mais cette hypothèse permet aussi de trouver une réponse au problème que veut résoudre Rawls en exigeant que la raison publique fasse l'objet d'une allégeance morale, c'est-à-dire le problème de la stabilité des institutions démocratiques.

Parce que, conformèment à cette hypothèse, des individus libéraux entretiennent des conceptions spécifiquement politiques $\mathrm{du}$ bien, parce que ces conceptions du bien politique sont relativement indépendantes des autres conceptions morales qu'entretiennent aussi

21. Lintuition de base ici est que lorsque les valeurs morales sont articulées dans des doctrines consistantes (des systèmes), il suffit d'un désaccord sur une seule valeur (une partie du système) pour être en désaccord avec le tout. Si les individus sont pluriels dans leurs systèmes de valeurs, ils peuvent ètre en désaccord sur des valeurs qu'ils tiennent pour pertinentes dans un domaine donné et s'entendre néanmoins sur les valeurs morales pertinentes dans le domaine politique. C'est dans ce sens que l'étendue des conceptions compréhensives chez Rawls fait croitre les risques d'incommensurabilité entre les croyances individuelles et fait décroître, par conséquent, le nombre des " idées communes *. 
les citoyens, l'allégeance morale à la raison publique ne fait appel qu'à des conceptions du bien politique. Les conceptions morales non politiques ne risquent pas de faire obstacle à la pratique de la raison publique; la "pratique citoyenne* ne risque pas de rester lettre morte.

D'autre part, parce que les normes de la raison publique définissent la pratique citoyenne comme une pratique en partie morale, parce que cette pratique fait vraisemblablement appel à un plus grand nombre de valeurs morales communes et parce que celles-ci n'entrent pas en contradiction, pour un individu libéral, avec les valeurs qu'il juge pertinentes dans d'autres domaines, la pratique citoyenne ne risque pas de générer le scepticisme moral. Pour les mèmes raisons, la raison publique ne risque pas non plus de se substituer aux autres conceptions du bien qu'entretiennent les citoyens et de devenir elle-mème une conception compréhensive.

Pour accepter de se faire l'exécuteur de la raison publique, le citoyen n'a pas à voir la société comme un bien instrumental, ni comme une partie d'une conception compréhensive ; il accepte de se faire l'exécuteur de la raison publique parce qu'elle est publique et qu'elle peut conséquemment garantir l'endurance de ce type d'organisation politique qui correspond à sa conception de la vie bonne dans le domaine politique.

Plusieurs critiques ont reproché à Rawls de mettre de l'avant une conception du raisonnable qui présuppose en fait la raison libérale. Rawls ne s'en est jamais caché : les raisons susceptibles de rendre raisonnables les arrangements politiques comptent pour des raisons auprès de ceux à qui il importe d'agir pour des raisons et qui sont eux-mêmes disposés à justifier leurs décisions sur la base de raisons accessibles à autrui. Ni l'intolérance, ni le dogmatisme, ni la cœercition, ni le fanatisme, ni la démagogie, ni la tyrannie ne sont raisonnables. Le libêralisme colore aussi la nature même des raisons admissibles chez Rawls. Les libertés civiles et politiques, l'égalité des chances, la valeur du pluralisme et de la participation démocratique sont des raisons accessibles au libéral, mais qui ne le seraient pas nécessairement, ou mème probablement pas, à celui qui ne souscrit ni à l'esprit ni à la lettre du libéralisme.

On a reproché à Rawls de mettre de l'avant une conception de la justice taillée sur mesure pour le libéral, une conception qui ne peut paraitre acceptable qu'aux yeux de celui qui souscrit déjà au libéralisme $e^{22}$. Rawls n'a jamais nié que telle était son intention; le citoyen idéal, de mème que celui dont on solliciterait l'avis, dans une société actuelle, au sujet de la conception rawlsienne de la justice, celui dont Rawls suppose qu'il donnerait son accord à cette même conception, est le citoyen d'une démocratie constitutionnelle libérale, qui connait bien les institutions et les modes de fonctionnement diun

22. Cette objection, tout comme la précédente, s'applique aussi d'une façon générale au libéralisme. Différentes versions de cette critique ont été formulées, par exemple, par Thomas Nagel dans "Moral Conflict and Political Legitimacy ", Philosophy and Public Affairs 16, p. 215-240 et par J. Hampton dans "Should Political Philosophy be done without Metaphysics ". 
qui connait bien les institutions et les modes de fonctionnement d'un Etat libéral contemporain. Et telles doivent ètre, selon Rawls, les limites de la philosophie politique. Une conception politique de la justice ne doit pas aspirer à l'universalité ; elle est une conception pour une société donnée, parvenue à un point donné de son évolution.

Mais l'individu dont l'allégeance au libéralisme politique dépend d'une conception compréhensive du bien est-il toujours le citoyen, idéal ou actuel, d'une démocratie libérale contemporaine parvenue au degré d'évolution que nous lui connaissons maintenant ? L'idée de conception compréhensive n'est plus à notre époque, comme elle pouvait l'ètre au temps de John Stuart Mill, une idée libérale ; elle ne s'accorde ni avec la prudence que le libéral contemporain pratique à l'endroit des doctrines ou des vérités supposément établies, ni avec l'autonomie qu'il reconnait aux différentes sphères de son activité, ni avec la liberté qu'il se réserve de juger et de décider en tenant compte des circonstances et des options disponibles. Le citoyen d'une démocratie constitutionnelle contemporaine qui entretiendrait une conception compréhensive, de quelque nature que ce soit, n'aurait pas intériorisé les principes libéraux de la tolérance et du pluralisme. Il ne les appliquerait pas à ses propres croyances puisque, par définition, il tiendrait certaines de celles-ci pour valides dans tous les secteurs de son activité. Il pourrait se plier aux exigences d'une raison publique libérale, si telle était la raison dominante dans sa sociêté, mais, comme nous l'avons vu, il n'en serait jamais le défenseur.

Si les arguments avancés au cours de cet article sont fondés, force nous est de conclure qu'en faisant dépendre l'allégeance au libéralisme de conceptions morales compréhensives, Rawls fait $\mathrm{du}$ libéralisme politique un idéal non seulement inaccessible, mais aussi inacceptable du point de vue d'une conception libérale de la moralité et de la manière dont elle intervient dans la conduite de la vie des citoyens d'une démocratie libérale contemporaine. En faisant dépendre l'allégeance au libéralisme politique de conceptions morales qui vont à l'encontre de l'esprit du libéralisme contemporain, Rawls pourrait bien outrepasser les limites qu'il s'est lui-mème assignées et que ne devrait jamais outrepasser, selon lui, la philosophie politique. Si une conception politique de la justice est une conception pour une société donnée parvenue à un point donné de son évolution, alors la conception de la justice de Rawls n'est pas une conception politique : elle est, au mieux, une conception morale pour une société hypothétique, susceptible de répondre aux attentes de personnes morales tout aussi hypothétiques, entièrement caractérisées par leur volonté d'entretenir et de mener à terme une conception compréhensive du bien. Si une société pareillement constituée devait voir le jour, elle ne souscrirait pas, comme nous croyons l'avoir montré ici, à une raison publique libérale. 


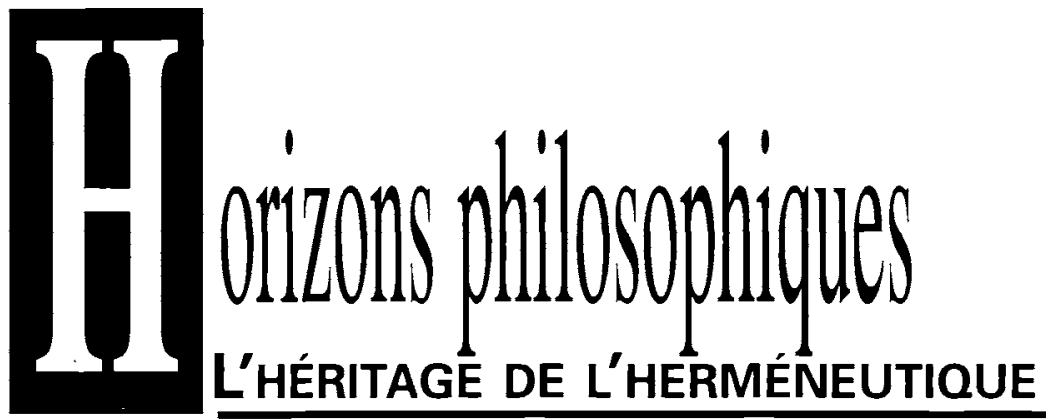

Liminaire

Après I'herméneutique - Analyse et interprétation des traces

et des auvres, Jean Molino.

Faits et interprétations en musicologie, Jean-Jacques Nattiez ..........................33

Théorie des préjugés selon Descartes et Gadamer, Donald Ipperciel ..............43

Un problème d'appropriation : Schleiermacher entre

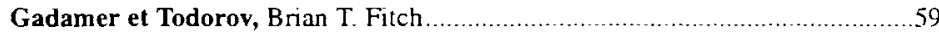

L'état idéal de finitude chez Gadamer, Alain Beaulieu .............................75

Husserl et le Ichprinzip • Le tournant en question, Éric Paquette ..................... 91

Christoph Menke : herméneutique, déconstruction

et raison dans le problème de l'expérience esthétique, Michel Ratté ....

Sujet(s) à interprétation(s) : sur la relative transparence

d'une photographie positiviste, Jean Lauzon

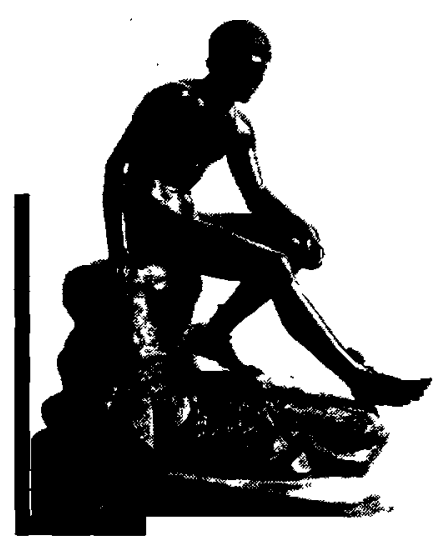

Compte-rendus :

Nycole Paquin et al,

De l'interprétation en arts visuels, Jean Lauzon

Paul Riccur, La critique et la conviction; Réflexion faite,

autobiographie intellectuelle, Marc Chabot.

Daniel Pinkas, La matérialité de l'esprit, Luc Abraham.

Gilbert Hottois, Entre symboles \& technosciences,

Karine R. Damar Singh

Marc Jeannerod, De la physiologie mentale. Histoire des relations

entre la biologie et la psychologie, Claude Lagadec

Tobie Nathan et Lucien Hounkpatin, La parole de la forêt initiale, Jean-Claude Muller ........................................................... 153

Ouvrages reçus

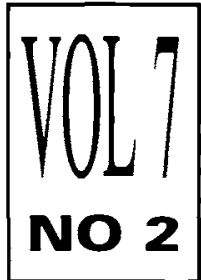

Abonnements :

Renée Asselin

945, chemin de Chambly,

Longueuil (Québec) J4H 3M6

Tél : (514) 679-2630, poste 487

Télécopieur : (514) 677-2945 